\title{
Pilots' Visual Scan Patterns and Attention Distribution during the Pursuit of a Dynamic Target
}

Chung-San Yu ${ }^{1}$, Eric Min-yang Wang ${ }^{1}$, Wen-Chin Li², Graham Braithwaite ${ }^{2}$ \& Matthew Greaves ${ }^{2}$

${ }^{1}$ Department of Industrial Engineering and Engineering Management, National Tsing Hua University

101 Sec. 2, Kuang-Fu Rd. Engineering Building 1, RM 711, Hsinchu City 30013, Taiwan, Republic of China

${ }^{2}$ Safety and Accident Investigation Centre, Cranfield University Cranfield, Beds MK43 OAL, United Kingdom

Tel: +886 (3) 5742929 (Chung-San Yu, PhD Candidate)

+886 (3) 5742649 (Eric Min-yang Wang, PhD)

+44 (1234) 758527 (Wen-Chin Li, PhD)

+44 (1234) 754252 (Graham Braithwaite, PhD)

+44 (1234) 754243 (Matthew Greaves, PhD)

Fax: $\quad+44(1234) 758527$

E-mail: mountainsyudyahoo.com (C-S. Yu)

E-mail: mywangericegmail.com (E. M-Y. Wang)

E-mail: wenchin.li@cranfield.ac.uk (W-C. Li)

E-mail: g.r.braithwaitedcranfield.ac.uk (G. Braithwaite)

E-mail: m.j.greaves@cranfield.ac.uk (M. Greaves)

Running Head: Pilots' Attention Distribution Aiming a Dynamic Target Manuscript metrics

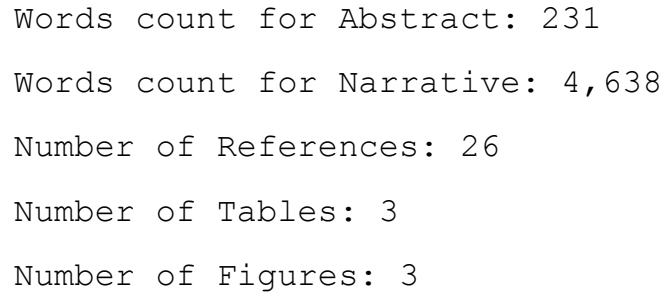

Published by Aerospace Medical Association. This is the Author Accepted Manuscript. 


\section{ABSTRACT}

Introduction: The current research is investigating pilots' visual scan patterns in order to assess attention distribution during air-to-air manoeuvers. Method: A total of thirty qualified mission-ready fighter pilots participated in this research. Eye movement data were collected by a portable head-mounted eye-tracking device, combined with a jet fighter simulator. To complete the task, pilots have to search for, pursue, and lock-on a moving target whilst performing air-to-air tasks. Results: There were significant differences in pilots' saccade duration (msec) in three operating phases including searching ( $\mathrm{M}=241, \mathrm{SD}=332)$, pursuing ( $\mathrm{M}=311, \mathrm{SD}=392)$, and lock-on (M=191, SD=226). Also, there were significant differences in pilots' pupil sizes (pixel ${ }^{2}$ ) of which lock-on phase was the largest $(M=27237, S D=6457)$, followed by pursuing ( $M=26232, S D=6070)$, then searching (M=25858, $S D=6137)$. Furthermore, there were significant differences between expert and novice pilots on the percentage of fixation on the HUD, time spent looking outside the cockpit, and the performance of situational awareness (SA). Discussion: Experienced pilots have better SA performance and paid more attention to the HUD but focused less outside the cockpit when compared with novice pilots. Furthermore, pilots with better SA performance exhibited a smaller pupil size during the operational phase of lock-on whilst pursuing a dynamic target. Understanding pilots' visual scan patterns and attention distribution are beneficial to the design of interface displays in the cockpit and in developing human factors training syllabi to improve safety of flight operations.

Keywords: aviation safety, pupil size, saccade duration, situational awareness, training evaluation 


\section{INTRODUCTION}

The advanced technologies of Head-Up Displays (HUD) have provided large amounts of information rapidly and precisely to improve pilots' situational awareness (SA) and facilitate a successful sortie. Pilots process information relying on the perceived stimulus from the visual environment, which might potentially lead to confusion and perceptual illusions in certain situations (18). Information processed by pilots is mostly acquired by visual scans of interior and exterior of the cockpit, and majority of pilot errors in flight operations resulted from poor situational awareness (11). The visual scan pattern is a precursor to initiating the cognitive process, and information from the eye movements within cockpit are directly connected with a pilot's attention allocation (16).

The pattern of eye movement is one of the methods for assessing pilots' cognitive processes based on real-time physiological measure (7). Eye-tracking devices have been applying to human-computer interaction domains for a long time, such as cockpit display design (10); display design for air traffic controllers (1); displays of interfaces for uninhabited aerial vehicles (24); and design of control rooms of nuclear power plants (6). In general, an individual spends more time looking at important or interesting objects in the environment. The length of fixation duration can reflect difficulty in extracting information, and the number of fixations indicates the importance of the areas of interest (AOIs) (13). Also, the phenomenon of tunnel attention could be observed by the concurrence of an excessive long fixation duration dwelled on a specific area, reduced saccades (10), and decreased scanning frequency on the display 
interfaces (15). Research on the differences in visual time distribution has suggested that experts spend more time looking at thematically relevant elements of a task, while novices spend more time on the salient stimuli (2).

Pilots' attention distributions play a central role in cognitive processing, and eye movements may serve as a window into the visual scan pattern for acquiring SA, which is a precursor for pilots' aeronautical decision-making (ADM). Lack of visual attention is an indicator of missing $\mathrm{SA}$, which is a known contributing factor in aviation accidents (17). Although there is a debate concerning a 'bottom-up' or 'top-down' approach to visual attention in the eye-tracking research, it was observed that pilots integrated both bottom-up and top-down visual processes based on their experience and salience of information during tactical operations (25). The bottom-up eye movement is a stimulus-based visual process. The salient cues attract the pilot's gaze to pay attention (such as an activated warning light) by visual scan to perceive the stimulus. The level-1 of SA is a bottom-up approach for perceiving the stimulus of activated warning light whilst level-2 and level-3 of SA are top-down visual processes for understanding the stimulus by cross-checking the information, then projecting the probable course of action in the near future (3). The analysis of frame-by-frame eye tracking data can proceed using both a top-down approach based on design hypotheses; and a bottom-up approach based on observation of the data without predefined theories relating eye movements to cognitive activity (5).

Expert pilots have been shown to have a longer duration of focus on relevant cues when a warning light was present (22). Furthermore, 
expert pilots are able to attend quickly to relevant indicators for required information when making decisions, with a pattern of more fixations and shorter fixation duration during landing operations (12). Therefore, it was suggested that fixation numbers and fixation duration focused on a certain AOIs might indicate where attention is allocated. The numbers of fixations distributed and fixation duration on relevant AOIs can be not only an effective indicator of pilot's expertise level but can also be closely related to a pilot's situational awareness (25). Military pilots operating aircraft during tactical manoeuvers have to identify rapidly and precisely where, or how, to move to the most appropriate position based on the relative dynamic information of a moving target, as pilots have to assess potential risks of intended tactical manoeuvers to gain the most advantageous dynamic positions by projecting the trajectory movement in the near future. Experienced pilots frequently switch their attention more efficiently to search and acquire relevant information compared with novices, as extensive visual surveys are critical for getting attention to ensure that saccades land on the selected object in a timely manner (14). Therefore, effective saccades play an important role in pursuing a moving target during air-to-air manoeuvers. It appears that saccades might be associated with pilot's attention shifting for pursuing a dynamic target, as saccade duration is the total time to make a saccade and the saccade velocity is how fast the eyes move between fixations (19).

The definition of $\mathrm{SA}$ is the perception of the elements in the environment within a volume of time and space (level-1), the comprehension of their meaning (level-2), and the projection of their 
status in the future (level-3) (3). In this definition the higher level of SA, which is a projection of future status by cognitive processing, depends on the lower level of SA, which is the perceived information. As most information in the cockpit is presented visually and over 75\% of pilots errors are related to perceptual failures (11), it is very important to understand pilots' visual information processing and eye movement patterns since they underpin SA performance in flight operations. Military pilots have to detect, recognize, and identify a foe via visual scan and displays of radar for conducting target acquisition, target tracking, weapon release and in order to fly-out safely. Pilots' eye movements in response to tracking a dynamic target usually represent a fast linear readout of direction and speed-tuned activities of cognitive processing. Selective attention results in the enhancement of relevant information and suppression of irrelevant information. For example, the time interval from the ending time of the last fixation on the Safe Check Switch (SCS: a three-way toggle switch for ordnance safety) to the starting time of the first fixation on the moving target is a critical saccadic interval. It might be linked with attention distribution, which could be the factor impacting a pilot's SA performance in tracking a moving target (20) . Although pilots can look in one direction and attend to another, covert and overt attention are often aligned in space, making eye movements a useful means to assess visual attention during searching (26).

Pupil dilation is a quick response not only to changes in the brightness of the visual environment, but also of a person's cognitive workload while performing a visual task such as tracking a moving target. Pupil size is an important indicator to understand an 
operator's visual attention and workload, and it has been used to assess training effectiveness (23) and to explore a pilot's mental process, cognitive workload and visual attention objectively while performing a flight task (15). In addition, pupil diameter changes were significantly higher when subjects were performing well on the auditory task compared with subjects performing poorly (1). Pilots have to estimate the trajectory movements and relative approaching speed of a target, and use this information to decide where and how to move to the best intercept position. There have been several accidents involving air-to-air manoeuvers that resulted in Controlled Flight into Terrain (CFIT) as pilots focused on manoeuvering and overlooked the relative position of the aircraft and terrain. Causes included pursuing a foe into cloud, inducing spatial disorientation, or ineffective visual scan resulting in the loss of situational awareness and flying below the safe altitude limit (17).

Eye-tracking techniques are efficient in identifying attentional distribution and assessing cognitive countermeasures. There is an increasing need to study pilots' attention distribution, selective attention and attention shift during the pursuit of a dynamic target in order to improve aviation safety. Therefore, the objectives of the study were (1) to investigate pilot's scan pattern among different AOIs during the pursuit of a dynamic target, (2) to evaluate pilots' saccades and attention shift during the tasks, and (3) to measure the relationship between pupil size and SA performance during pursuing a dynamic target. 
METHODS

Subjects

A total of thirty mission-ready fighter pilots participated in this research. The ages of subjects ranged between 26 and 51 years old ( $M=29$, $\mathrm{SD}=6)$. Their total flying experiences varied between 310 and 2,920 hours (M=844, SD=720). The subjects were categorized into the novice group ( $\mathrm{n}=15$ ) with total flight hours below 550 (M=370, SD=68), and the expert group ( $\mathrm{n}=15)$ with equal to and over 550 flight hours (M=1319, $\mathrm{SD}=766$ ). The threshold between expert and novice pilots is 550 flight hours, as it is the milestone for fighter pilots to be the leader of a two-ship tactical formation. All of the subjects are volunteers and were informed that there was no incentive to secure participation; subjects had the right to cease the experiment and withdraw provided information without any reason, and the storage of obtained data was in accordance with the Data Protection Act. The treatment of subjects was approved by the Research Ethics Regulations of National Tsing Hua University.

Equipment

1. Flight Simulator: The simulator is equipped with a 2-D and 1:1 image projected on the 5-metre wide and 3-metre high screen. It utilizes an actual cockpit with identical display panels, layout and controls to those in the actual fighter, and is capable of supporting pilots' tactical operational training by providing a realistic representation of the combat mission. The instructor can install scenarios and observe the trainee pilot's performance via a three-screen console. The information display on the HUD indicates 
target's relative position through icons, letters, numbers, lines, and even figures. The Integrated Control Panel (ICP) is an interface like a keyboard for keying navigation and communication data, which is composed of 18 rectangular buttons, 9 circular knobs and some toggle switches. Right Multiple Function Display (RMFD) provides the information that the pilot keys in over the ICP, which is illustrated with letters and numbers. Left Multiple Function Display (LMFD) shows the radar information regarding the target and terrain through a digital map, lines and numbers. The foe's location, altitude, attitude, speed and heading which appeared at the start-point of scenario are fixed until the target is pursued by the interceptor. Basically, the behavior of the foe was programmed by the central computer to manoeuver with appropriate G-force to make its escape to avoiding lock-on by the interceptor.

2. Scenario of Simulator: The scenario of this experiment is an air-to-air manoeuver to analyze pilots' visual scanning shifts to search, pursue and lock-on a dynamic target. The altitude of the interceptor during patrol was 20,000 feet with a cruise speed of 300 knots indicated airspeed (KIAS) and a heading of $050^{\circ}$ with weather conditions of 7-mile visibility and scattered clouds. A foe unexpectedly appeared at the same altitude on a heading of $090^{\circ}$ with 300 KIAS of airspeed (figure 1). The subjects have to search the airspace for the target and intercept the target immediately by tactical manoeuvers. However, the target would change its heading, altitude and speed to escape from the interceptor's pursuit until the interceptor pressed the trigger and completed the task. At the same time, a senior instructor pilot (IP) would mark if the pilot terminated 
the target or not. In this study, three phases of visual behaviors were analyzed: searching for the target with eye contact (searching), pursuing for aiming (pursuing), and lock-on to the dynamic target (lock-on). In addition, the generator malfunction light on the Warning Light Panel (WLP) would illuminate unexpectedly during the phase of lock-on. This is in order to evaluate pilots' SA performance.

3. Eye Tracking Device: A mobile head-mounted eye tracker designed by Applied Science Laboratory (ASL Series 4000) was used to collect pilots' eye movement data. The eye tracker is a light (76g) and portable device, and it is easy for subjects to move their head without any limitations during the air-to-air combat scenario. The pattern of eye movements and the related data were collected by a Digital Video Cassette Recorder (DVCR) and transferred to computer for further analysis. The sampling frequency of this device is $30 \mathrm{~Hz}$, which means a one-second eye movement is captured in 30 frames. The definition of a fixation in the present research is three gaze points occurring within an area of 10 by 10 pixels with a dwell time (the time spent per glance at an area or instrument) over $200 \mathrm{msec}$. There were five AOIs set up to observe subjects' eye movement data during the air-to-air task. Those AOIs were selected by consultation with the chief training instructor as the most important elements in performing air combat manoeuvers. Those AOIs could provide the most vital information to complete the mission. The AOIs are detailed as follows, AOI-1: HUD; AOI-2: ICP; AOI-3: RMFD; AOI-4: LMFD; and AOI-5: Outside cockpit. 
[Figure 1 here]

Research Design

All subjects undertook the following procedures; (1) completed the demographical data on the performance evaluation form including rank, age, qualifications, type ratings and total flight hours, followed by reading the description of research ethics (5-7 minutes); (2) a short briefing to explain the purpose of the study and introduce the air-to-air scenario (5-10 minutes); (3) calibrating the eye tracking device by using three points distributed around the cockpit display panels and screen (10-20 minutes); (4) subjects performed the air-to-air task (2-3 minutes); (5) a debrief collected subjects' feedback and comments (5-10 minutes). In total, approximately 50 minutes was required for each subject to complete the experiment.

Based on the context of air-to-air manoeuvers, the main operational phases were defined as Searching, Pursuing and Lock-on for pick-off. To standardize the processes of data analysis, eye movement data was only counted for 15 seconds which comprised above three phases of tactical manoeuvers, as instructor pilot observed all subjects' performance and suggested that the phase of lock-on for pick-off is the most critical phase to terminate the foe. Therefore, starting from the point at which the foe was terminated the analysis tracked back through the three phases of lock-on for pick-off ( 5 seconds), pursuing target (5 seconds), and the searching phase (5 seconds). Those 15 seconds are the most important in terms of cognitive processes for military pilots performing an air-to-air mission. The total time of measurement for performing the task and the fixation duration should be considered concurrently. It is obvious that the longer the total 
time of measurement, the higher the number of fixations that will be counted. Due to the varied time frames for each pilot in performing the air-to-air mission (between 28 and 140 seconds) in the present study, standardizing the processes of data analysis is necessary. Pilots' eye movement data were analyzed by following dimensions: percentage of fixation; average fixation duration; pupil size and saccades occurring within AOIs. The unexpected event of a generator malfunction warning was activated by the IP during pilots pursuing the target. If the pilot called 'generator out' and pressed the master caution light, the IP would mark the pilot's SA performance as 'good $\mathrm{SA}^{\prime}$; if pilot didn't press the master caution would be marked as 'poor $\mathrm{SA}^{\prime}$. The current study adopted the embedded task measures to evaluate pilot's SA performance to avoid interrupting ongoing tasks.

\section{RESULTS}

Subjects' eye movement data described by percentage of fixation and average fixation duration among five AOIs are shown as table I. The 'percentage of fixation' is proportional data, therefore, it is necessary to perform an arcsine transformation before conducting analysis of variance (8). Significant effects among five AOIs were observed in terms of percentage of fixation during air-to-air combat, $\mathrm{F}(4,145)=164.35, p<.001, \eta 2 p=.85$. Further comparisons by post-hoc Bonferroni adjusted tests showed that AOI-5: Outside of cockpit (53.1\%) has a significantly higher percentage of fixation than AOI-2: ICP (2.5\%), AOI-3: RMFD (0.7\%) and AOI-4: LMFD (1.3\%); and AOI-1: HUD (35.8\%) has significantly higher percentage of fixation than ICP, RMFD and LMFD. Furthermore, there were significant differences in pilots' 
average fixation duration among the five different AOIs, F (4, 145) $=85.74, p<.001, \eta 2 \rho=.75$. Further comparisons by post-hoc Bonferroni adjusted tests showed that Outside of cockpit (460 msec) has significantly longer average fixation duration than HUD (457 msec), ICP (98 $\mathrm{msec}), \operatorname{RMFD}(34 \mathrm{msec}$ ) and LMFD (59 $\mathrm{msec})$; and HUD has significantly longer fixation durations than ICP, RMFD and LMFD.

[Table I here]

It is important to investigate the pupil size, saccade duration and saccade velocity, as saccades represent the mechanisms of fixation and rapid eye movement. The parameters of pupil size, average saccade duration and saccade velocity were analyzed by three operational phases of the air-to-air task; searching, pursuing, and lock-on. There were significant differences between pilots' pupil size in three operating phases, $F(2,87)=15.30, p<.001, \eta 2 p=.35$. Further comparisons by post-hoc Bonferroni adjusted tests showed that pilots' pupil size in the phase of lock-on (27237 pixel $^{2}$ ) was significantly larger than in pursuing (26232 $\mathrm{pixel}^{2}$ ) and in searching phases (25858 pixel $\left.{ }^{2}\right)$. Furthermore, there were significant differences in pilots' average saccade duration at the three operational phases, F $(2,87)$ $=6.43, p<.005, \eta 2 \rho=.18$. Further comparisons by post-hoc Bonferroni adjusted tests showed that pilots' average saccade duration at the phase of pursuing (311 $\mathrm{msec}$ ) was significantly longer than at searching (241 msec) and at lock-on (191 msec). However, there were no significant differences in pilots' average saccade velocity at the three operating phases, $\mathrm{F}(2,87)=.36, p>.05, \eta 2 p=.01$. 
[Table II here]

The differences of SA performance between experienced pilots and novice pilots by Chi-square test are shown as table II. Significance was observed in pilots' SA performance $\left(\chi^{2}=6.65, p<.05\right)$ between experts and novice pilots. It showed that $76.9 \%$ of experienced pilots and $23.1 \%$ of novice pilots could identify the activated warning light in highly demanding tactical combat manoeuvers. Furthermore, table III shows significant differences in percentage of fixation between the experienced and novice pilots on the HUD ( $t=3.78, p<.005, d=1.38$ ) and Outside of cockpit $(t=-4.12, p<.001, d=1.50)$. Experienced pilots have more fixations on the HUD (44.1\%) and fewer fixations outside of the cockpit (44.8\%) compared with novice pilots (HUD: 27.5\%; outside of cockpit: $61.5 \%$ ). To assess pilots' attention distribution and attention shift at the phase of lock-on, the two indicators 'length of duration on Safe Check Switch (SCS)' and 'interval from SCS to re-fixating the target' were evaluated. There were significant difference in the length of duration on $\operatorname{SCS}(t=4.42, p<.001, d=1.62$ ) and in time interval from SCS to re-fixating the target $(t=-2.60, p<.05$, $d=0.95)$. Experienced pilots spent more time $(605.1 \mathrm{msec})$ on SCS than novice pilots (388.3 $\mathrm{msec})$. However, experienced pilots spent significantly less time (398.1 $\mathrm{msec})$ from SCS to re-fixating the target compared with novice pilots (1185.5 msec). 


\section{DISCUSSION}

To search for a moving target during air-to-air manoeuvers, pilots have to divide attention, use selective attention, scan airspace and cockpit instruments in order to achieve situational awareness and conduct aeronautical decision-making in time (17). On the other hand, pilots may lose SA as they focus on pursuing the dynamic target and enter fatal zones if their fixations and attention were not directed to appropriate AOIs. In addition, pilots may be distracted and suffer mode confusion due to an unexpected system malfunction, which may limit a timely response in an emergency.

Table I indicates that the information captured by pilots' visual scans from the HUD and Outside of Cockpit are critical to conducting time-limited tactical manoeuvers for precisely tracking and aiming at a moving target. In addition, table III shows that there are significant differences of percentage of fixation on the HUD and Outside of Cockpit AOIs between experts and novice pilots during air-to-air manoeuvers. Military pilots have to shifting their attention between the information provided by the HUD and the movements of a dynamic target precisely to perform the tactical manoeuvers effectively. The findings of pilots' percentage of fixation and average fixation duration in the present study confirmed that pilots pursuing a moving target have to filter and evaluate the perceived cues from the HUD and trajectory movement of a foe outside of cockpit. The cognitive processes of attention distribution and selective attention are based on pilots' expectations, knowledge and experience (15). Therefore, pilots not only pay attention to the most salient stimulus (symbol on the HUD) but also shift their attention 
simultaneously to the main priority, which is the manoeuvers for tracking the target's unpredicted tactical movements.

The eye movement patterns shown by table I indicate that the highest percentage of fixation was outside of the cockpit. The results of the present study are different from the previous study (25), which proposed the highest percentage of fixation allocated on the HUD (59.92\%) followed by Outside of Cockpit (39.18\%). This difference is due to the context of the task between pursuing a dynamic target (air-to-air task) and stationary target (air-to-surface task). It is consistent with the findings of pilots who did not employ a standardized scanning pattern, but monitored their in-flight situation based on expectations associated with specific flight contexts (21). Although the average fixation duration on the HUD and the Outside of Cockpit are not much different - a difference of $3 \mathrm{msec}$ (457 and 460 msec respectively) - the phenomenon of longer fixation duration focused on the certain locations might indicate that the information coming from those AOIs is critical to the operation and in need of more attention (13).

\footnotetext{
[Figure 2 here]

[Figure 3 here]
}

\footnotetext{
According to figure 2(a), there were significant differences in pilots' saccade duration between the three operational phases. The shortest saccade duration was at the phase of lock-on followed by searching then pursuing. Figure 3 indicates different trends in saccade duration between experienced pilots and novice pilots in the
} 
three operational phases. Except for the phase of searching, expert pilots show longer saccade duration than novice pilots in the phases of pursuing and lock-on. Saccade duration is the total time to make a saccade between fixations which may reflect the path of attention shift (15). During the searching phase expert pilots made a saccade taking significantly shorter time (211 msec) to shift their attention than novice pilots (271 $\mathrm{msec})$. It reveals that expert pilots can accurately identify the cues of a moving target on the HUD and grasp the image of foe by shifting attention. It was found that while pursuing the moving target, the saccade was related to the trajectory of the target's movements. When the target was captured by eye-contact at the phase of pursuing, expert pilots deployed significantly longer duration (347 $\mathrm{msec})$ than novice $(275 \mathrm{msec})$ to distribute wider attention shift for monitoring the holistic situation. On the other side, novice pilots might narrow down their attention by only conducting the tactical manoeuver of pursuing for aiming at the moving target since they were only focused on the task performance of pursuing the foe and rather than shifting their attention to the holistic operating environment. It is possible that this is the reason only 23.1\% of novice pilots perceived the warning light of generator malfunction and projected trouble-shooting processes for assessing SA performance (table II).

Although there were no significant differences in saccade velocity between expert and novice pilots across the three operational phases, the results reflect that the closer pilots were to the target, the faster their saccade velocity to shift attention during the dog-fight. The fastest saccade velocity occurred at the phase of lock-on, 
followed by searching, then pursuing. The attention shift to critical stimulus both on the HUD and from visual scan should simultaneously direct pilots' attention to the most important signals; this is crucial to successful execution of the task. However, the total time of measurement for performing the task and the fixations should be considered concurrently, since the longer period of measurement time is usually accompanied with more fixation points.

Pilots' visual scan patterns for attention distribution among AOIs and selective attention are a critical component in pursuing a dynamic target. However, the cognitive processes of selective attention related to prioritizing the information perceived is reinforced by pilot's knowledge and experience. Selective attention of pilots is important, as human being's perceptual system has limited processing capacity which enables pilots to constrain the selection of the appropriate incoming information and become aware of the presence of environmental changes (10). However, novice pilots' fixation distribution on the Outside of Cockpit is significantly higher than experienced pilots. The visual scan patterns show experienced pilots distributed their attention on seeking target-related information from the HUD and Outside of Cockpit almost equally. In comparison, novice pilots paid significantly more attention to the outside of Cockpit during air-to-air manoeuvers, which might suggest that their utilization of the HUD is less proficient than experienced pilots. In addition, quick distribution of attention between interior and exterior of cockpit might be the reason that higher percentage of experienced pilots showed good SA performance at the unexpected activation of the generator malfunction warning light. Pilots' mental 
status in such a critical phase was not only revealed with shorter saccade duration, but was also reflected in pilots' pupil size. Pupil size is influenced by illumination, and also by the difficulty and complexity of tasks in hand and the pilots' cognitive workload (4). Figure 2(b) indicates that the pilots' pupil size in the phase of lock-on $\left(27,237\right.$ pixel $\left.^{2}\right)$ is significantly higher than when pursuing $\left(26,232 \mathrm{pixel}^{2}\right)$ or searching $\left(25,858 \mathrm{pixel}^{2}\right)$. It reveals that pilots reach the most complicated situation for making decisions (to fire or not to fire) at the operational phase of 'lock-on for pick-off'. Therefore, this finding could justify the research design of the present study that the warning light for evaluating pilots' SA performance was designated to be activated at the phase of lock-on. Current research find that experienced pilots have shorter duration on weapon Safe Check during the operation of lock-on and shorter interval time from visual checking the weapon safe switch opened for tactical operations to re-fixating the target than the novice (table III). The application of an eye-tracker is appropriate to measure where pilot's visual attention is allocated (26). However, the main challenge of the eye-tracker is related to the retrospective analysis based on the eye movement data recorded by a near real-time approach. The problem of retrospective analysis is to find appropriate ways to interpret the data concerning human being's cognitive processing and behavior (9). The findings related to the differences between experts and novices of attention distribution on visual scan patterns for seeking information might be applied to the assessment of pilots' competence in pursuing moving target in the tactical manoeuvers. 


\section{CONCLUSION}

The findings of the current research into the pursuit of a moving target compared with the previous study of tracking a stationary target (25) indicate that pilots do not apply standardized visual scanning patterns, but rather they are based on situational requirements associated with specific operational contexts. Pilot's attention distribution seems to be closely related to level-1 (perception) of the three-level SA model (3), especially in searching for a moving target in open airspace. Thus, selective attention was mostly conducted after the target was pursued and lock-on, which corresponds to the level-2 (comprehension) and level-3 (projection) of the SA framework. Therefore, future application might have two directions; either to explore the interface design for maintaining operator's attention to improve situational awareness, such as Primary Flight Displays of airliners and the displays of ATC control panels; or develop training syllabi to increase operator's cognitive processes of visual scan for attention distribution. An eye-tracker is an appropriate device offering a non-intrusive approach to investigate in-flight visual attention and for analyzing pilots' cognitive processing, which can offset the weakness of traditional flight training. Specifically, the application of an eye-tracking device combined with a fighter simulator allows for the study of pilots' pupil size, saccades, fixations on AOIs and SA performance while operating air-to-air manoeuvers. Understanding pilots' visual scan pattern and attention distribution can have potential applications 
to improve the design of interfaces, and develop training to improve aviation safety. 


\section{REFERENCES}

1. Ahlstrom U, Friedman-Berg FJ. Using eye movement activity as a correlate of cognitive workload. Int J Ind Ergonom 2006; $36(7): 623-36$.

2. Carmichael A, Larson A, Gire E, Loschky L, Rebello NS. How does visual attention differ between experts and novices on physics problems? In: Proceedings of the Physics Education Research Conference; OR. Portland, USA; 2010: 93-6.

3. Endsley MR. Measurement of situation awareness in dynamic systems. Hum Factors 1995; 37: 65-84.

4. Gabay S, Pertzov Y, Henik A. Orienting of attention, pupil size, and the norepinephrine system. Atten Percept Psychophys 2011; 73: 123-29.

5. Goldberg JH, Stimson MJ, Lewenstein M, Scott N, Wichansky AM. Eye tracking in web search tasks: Design implications. In: Proceedings of the Eye Tracking Research and Applications Symposium; NY. New York, USA; 2002: 51-8.

6. Ha CH, Kim JH, Lee SJ, Seong PH. Investigation on relationship between information flow rate and mental workload of accident diagnosis tasks in NPPs. IEEE T Nucl Sci 2006; 53 (3) : $1450-59$.

7. Henderson JM. Human gaze control during real-world scene perception. Trends Cogn Sci 2003; 7(11): 498-504. 
8. Howell DC. Statistical methods for psychology, Belmont: Wadsworth; 2013: 346-52.

9. Jacob RJK, Karn KS. Eye tracking in human-computer interaction and usability research: Ready to deliver the promises (section commentary). Hyona J, Radach R, Deubel H. eds. The mind's eye: Cognitive and applied aspects of eye movement research. Amsterdam: North Holland; 2003: 573-605.

10. Johnson A, Proctor RW. Attention: theory and practice, London: Sage Publications, Inc.; 2004: 254-84.

11. Jones DG, Endsley MR. Sources of situation awareness error in aviation. Aviat Space Environ Med 1996; 67: 507-12.

12. Kasarskis P, Stehwien J, Hickox J, Aretz A, Wickens C. Comparison of expert and novice scan behaviors during VFR flight. In: Proceedings of the 11th International Symposium on Aviation Psychology; March 5-8, 2001; Ohio. Columbus, OH: Ohio State University, USA; 2001.

13. Kotval XP, Goldberg JH. Eye movements and interface components grouping: An evaluation method. In: Proceedings of the Human Factors Society 42nd Annual Meeting; CA. Santa Monica, USA; 1998: 486-490.

14. Kowler E. Attention and eye movements. Krauzlis R, ed. Encyclopaedia of Neuroscience, Amsterdam: North Holland; $2008: 605-16$.

15. Kowler E. Eye movements: The past 25 years. Vision Res 2011; 51: 
$1457-83$

16. Lavine RA, Sibert L, Gokturk M, Dickens B. Eye-tracking measures and human performance in a vigilance task. Aviat space Environ Med 2002; 73: 367-72.

17. Li WC, Harris D. A systems approach to training aeronautical decision-making: From identifying training needs to verifying training solutions. Aeronaut J 2007; 111: $267-79$.

18. Morgan-Warren PJ, Woodcock M. Visual aspects of advanced sensors and helmet-mounted displays. Aeronaut J 2014; 118: 155-68.

19. Rayner $\mathrm{K}$. Eye movements in reading and information processing: 20 years of research. Psychol Bull 1998; 124(3): 372-422.

20. Rognin L, Grimaud I, Hoffman E, Zeghal K. Assessing the impact of a new instruction on air traffic controller monitoring tasks. In: Proceedings of the International Conference on Human-Computer Interaction in Aeronautics; Toulouse, France; 2004: 197-203.

21. Sarter NB, Mumaw RJ, Wickens CD. Pilots' monitoring strategies and performance on automated flight decks: An empirical study combining behavioral and eye-tracking data. Hum Factors 2007; $48(3): 347-57$.

22. Schriver AT, Morrow DG, Wickens CD, Talleur DA. Expertise differences in attentional strategies related to pilot 
decision making. Hum Factors 2008; 50: 864-78.

23. Sibley C, Coyne1 J, Baldwin C. Pupil dilation as an index of learning. In: Proceedings of the Human Factors and Ergonomics Society Annual Meeting; NV. Las Vegas, USA; 2011 : $237-41$

24. Tvaryanas AP. Visual scan patterns during simulated control of an uninhabited aerial vehicle (UAV). Aviat Space Environ Med 2004; 75: 531-38.

25. Yu CS, Wang EM, Li WC, Braithwaite G. Pilots' visual scan patterns and situation awareness in light operations. Aviat space Environ Med 2014; 85: 708-14.

26. Zelinksy GJ. A theory of eye movements during target acquisition. Psychol Rev 2008; 115: 787-835. 
TABLE I

\begin{tabular}{lcccccc}
\hline \multirow{2}{*}{ Measures } & & \multicolumn{4}{c}{ AOIS } \\
\cline { 3 - 6 } & & HUD & ICP & RMFD & LMFD & OC \\
\hline Percentage of fixation & Mean & 35.8 & 2.5 & 0.7 & 1.3 & 53.1 \\
(arcsine values) & SD & 14.55 & 4.73 & 2.1 & 3.01 & 13.8 \\
Average fixation duration & Mean & 457 & 98 & 34 & 59 & 460 \\
(msec) & SD & 152 & 163 & 107 & 140 & 102 \\
\hline \hline
\end{tabular}

TABLE I. MEANS and STANDARD DEVIATIONS on the PERCENTAGE Of FIXATION and AVERAGE FIXATION DURATION among FIVE AOIs during AIR-TO-AIR COMBAT

AOI-1 : HEAD-UP DISPLAY (HUD) ; AOI-2: INTERGRATED CONTROL PANEL (ICP) ; AOI-3: RIGHT MULTIPLE FUNCTION DISPLAY (RMFD); AOI-4: LEFT MULTIPLE FUNCTION DISPLAY (LMFD); and AOI-5: OUTSIDE of COCKPIT (OC). 
TABLE II

\begin{tabular}{|c|c|c|c|c|c|c|}
\hline \multirow{2}{*}{ Groups } & \multirow{2}{*}{ Number } & \multicolumn{2}{|c|}{ SA performance } & Pearson & \multicolumn{2}{|c|}{ Chi-square } \\
\hline & & Poor & Good & $x^{2}$ & $d f$ & p-value \\
\hline Experienced & 15 & $\begin{array}{c}5 \\
(29.4 \%)\end{array}$ & $\begin{array}{c}10 \\
(76.9 \%)\end{array}$ & & & \\
\hline Novice & 15 & $\begin{array}{c}12 \\
(70.6 \%)\end{array}$ & $\begin{array}{c}3 \\
(23.1 \%)\end{array}$ & 6.652 & 1 & 0.01 \\
\hline
\end{tabular}

TABLE II. CHI-SQUARE Of SA PERFORMANCE between EXPERIENCED and NOVICE PILOTS 
TABLE III

\begin{tabular}{|c|c|c|c|c|c|c|c|c|}
\hline \multirow{2}{*}{ Variables } & \multirow{2}{*}{ Groups } & \multirow{2}{*}{ Mean } & \multirow{2}{*}{$S D$} & \multicolumn{5}{|c|}{ T-Test } \\
\hline & & & & $t$ & $d f$ & $p$ & $S E$ & Cohen's d \\
\hline \multirow{2}{*}{$\begin{array}{l}\text { Percentage of fixation } \\
\text { on the HUD (AOI-1) }\end{array}$} & Exp. & 44.1 & 10.1 & \multirow{2}{*}{3.784} & \multirow{2}{*}{28} & \multirow{2}{*}{0.001} & \multirow{2}{*}{6.25} & \multirow{2}{*}{1.384} \\
\hline & Nov. & 27.5 & 13.7 & & & & & \\
\hline \multirow{2}{*}{$\begin{array}{l}\text { Percentage of fixation } \\
\text { on OC (AOI-5) }\end{array}$} & Exp. & 44.8 & 9.6 & \multirow{2}{*}{-4.118} & \multirow{2}{*}{28} & \multirow{2}{*}{0.000} & \multirow{2}{*}{6.02} & \multirow{2}{*}{1.504} \\
\hline & Nov. & 61.5 & 12.3 & & & & & \\
\hline \multirow{2}{*}{$\begin{array}{l}\text { Length of duration on SCS } \\
\text { (msec) }\end{array}$} & Exp. & 605.1 & 134.3 & \multirow{2}{*}{4.424} & \multirow{2}{*}{28} & \multirow{2}{*}{0.000} & \multirow{2}{*}{48.99} & \multirow{2}{*}{1.619} \\
\hline & Nov. & 388.3 & 134.0 & & & & & \\
\hline \multirow{2}{*}{$\begin{array}{l}\text { Interval from SCS to } \\
\text { re-fixate the target } \\
\text { (msec) }\end{array}$} & Exp. & 398.1 & 301.9 & \multirow{2}{*}{-2.598} & \multirow{2}{*}{28} & \multirow{2}{*}{0.019} & \multirow{2}{*}{303.09} & \multirow{2}{*}{0.949} \\
\hline & Nov. & 1185.5 & 1134.4 & & & & & \\
\hline
\end{tabular}

TABLE III. MEANS and STANDARD DEVIATIONS Of VISUAL SCAN PATTERNS for EXPERIENCED and NOVICE PILOTS 
FIGURE 1

SA performance evaluation

(Malfunction warning light illuminated)

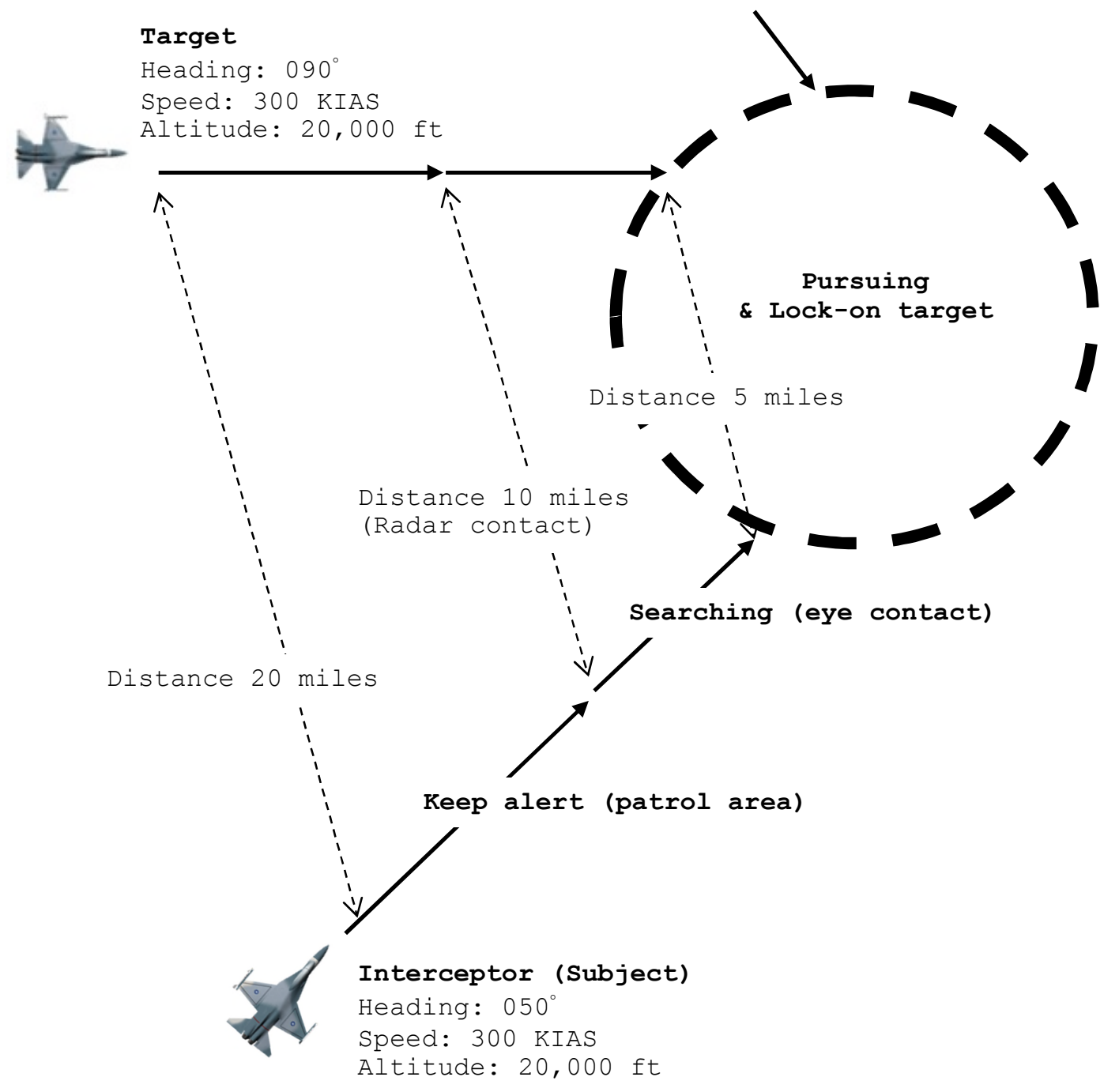

FIGURE 1. THE SCENARIO of AIR-TO-AIR MANEUVERS 
FIGURE 2

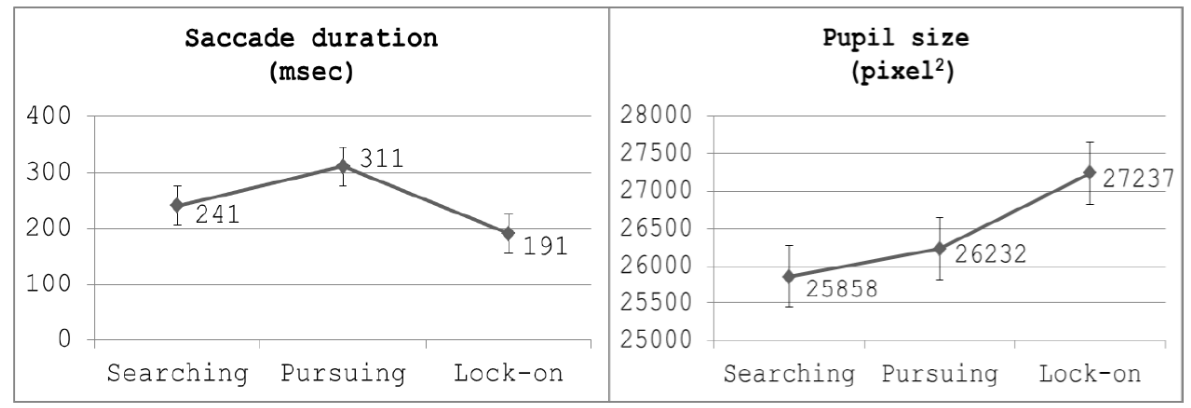

(a)

(b)

FIGURE 2. THE PATTERN Of PILOTS'SACCADE DURATION (msec) and PUPIL SIZE

$\left(\right.$ pixel $\left.^{2}\right)$ in THREE OPERATIONAL PHASES

FIGURE 3

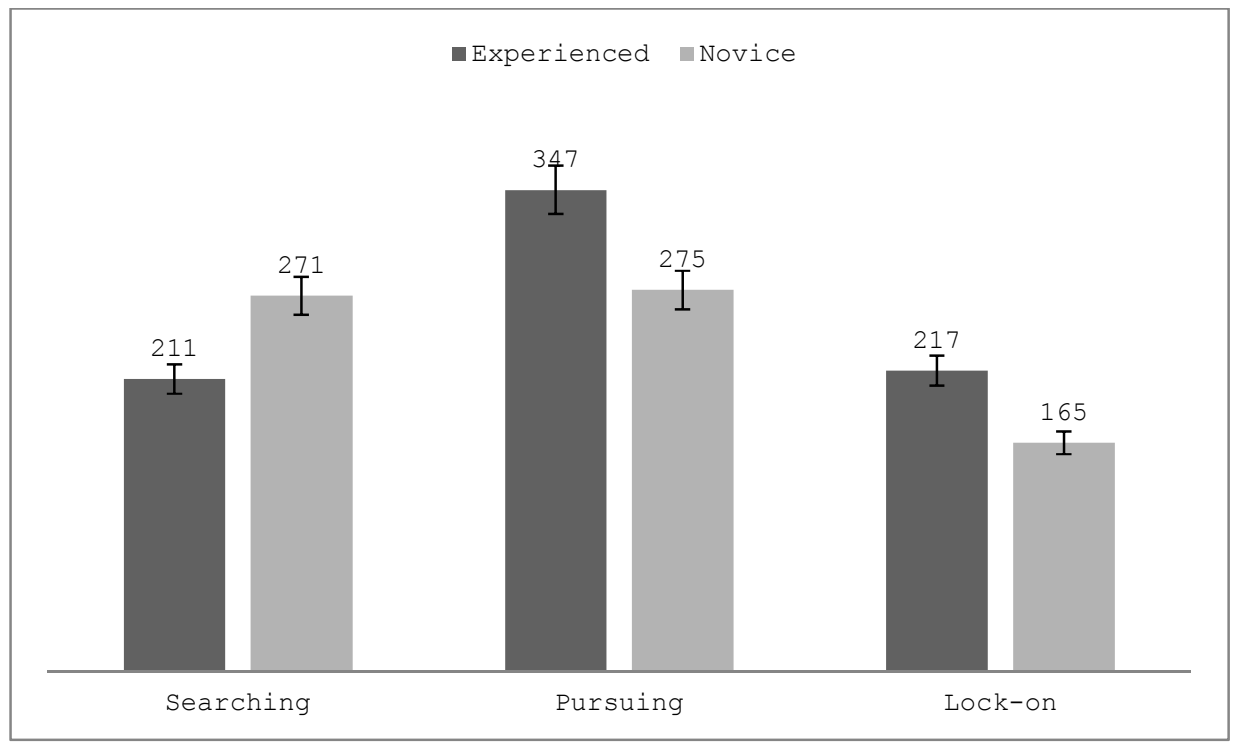

FIGURE 3. COMPARISON Of EXPERIENCED and NOVICE PILOTS' AVERAGE SACCADE DURATION in THREE OPERATIONAL PHASES (msec) 
2016-10-17

pÿPilots visual scan pattern and attention distribution during the pursuit of a dynamic target

\section{Yu, Chung-San}

Aerospace Medical Association

Chung-San Yu, Eric Min-yang Wang, Wen-Chin Li, Graham Braithwaite and Matthew Greaves. pÿPilots visual scan pattern and attention distribution during the pursuit of a dynamic target.

Aerospace Medicine and Human Performance, Volume 87, Number 1, January 2016, pp40-47 http://dx.doi.org/10.3357/AMHP.4209.2016

Downloaded from Cranfield Library Services E-Repository 